\title{
Holographic Entanglement Entropy in P-wave Superconductor Phase Transition
}

\author{
Rong-Gen Cai*, Song He ${ }^{\dagger}, \quad$ Li Li ${ }^{\ddagger}, \quad$ Yun-Long Zhang§ \\ State Key Laboratory of Theoretical Physics, \\ Institute of Theoretical Physics, Chinese Academy of Sciences, \\ P.O. Box 2735, Beijing 100190, People's Republic of China
}

April 26, 2012

\begin{abstract}
We investigate the behavior of entanglement entropy across the holographic p-wave superconductor phase transition in an Einstein-Yang-Mills theory with a negative cosmological constant. The holographic entanglement entropy is calculated for a strip geometry at AdS boundary. It is found that the entanglement entropy undergoes a dramatic change as we tune the ratio of the gravitational constant to the Yang-Mills coupling, and that the entanglement entropy does behave as the thermal entropy of the background black holes. That is, the entanglement entropy will show the feature of the second order or first order phase transition when the ratio is changed. It indicates that the entanglement entropy is a good probe to investigate the properties of the holographic phase transition.
\end{abstract}

\section{Introduction}

As a strong-weak duality, the AdS/CFT correspondence $[1,2,3]$ provides a powerful method for studying a strongly interacting system through its gravity dual which is weakly coupled. Especially, it has been used widely to model basic phenomena in condensed matter physics, such as superconductivity (superfluidity) [4], Nernst effect [5], and non-fermi liquid [6]. For more related studies, see, for example, Refs. [7, 8, 9] and references therein.

\footnotetext{
*E-mail: cairg@itp.ac.cn

${ }^{\dagger}$ E-mail: hesong@itp.ac.cn

†E-mail: liliphy@itp.ac.cn

§E-mail: zhangyl@itp.ac.cn
} 
The physical picture behind the holographic superconductor model is as follows. As the simplest concrete model, consider an Einstein-Maxwell-scalar field theory with a negative cosmological constant. At high enough temperature, the Reissner-Norström-AdS (RN-AdS) black hole with a trivial scalar field is stable. And the dual CFT is in a deconfined phase and describes a conductor phase. When one lowers the temperature of the black hole, the RN-AdS black hole becomes unstable, a new black hole solution with nontrivial scalar field is favored, which can describe a superconducting phase. The U(1) symmetry is spontaneously broken due to the nontrivial scalar field. The condensation of the scalar "hair" of the black hole gives a finite vacuum expectation value of the dual operator in the field theory side, which plays the role of order parameter in the holographic phase transition. The s-wave superconductor is described by the appearance of the scalar "hair" $[4,10,11,12]$, while the p-wave superconductor is characterized by the condensation of the vector "hair" $[13,14]$.

On the other hand, the entanglement entropy is expected to be a key quantity in our understanding some characterization of several aspects in many-body physics (see, for example, Refs. [15, 16]). For a given system, the entanglement entropy of subsystem with its complement is defined as the von Neumann entropy. In the spirit of AdS/CFT correspondence, a geometric proposal to compute the entanglement entropy has been presented in Ref. [17]. More precisely, consider a subsystem $\mathcal{A}$ of the total boundary system, the entanglement entropy of subsystem $\mathcal{A}$ with its complement is given by looking for the minimal area surface $\gamma_{\mathcal{A}}$ extended into the bulk with the same boundary $\partial \mathcal{A}$ of $\mathcal{A}$ (see Refs. [18, 19] for reviews)

$$
S_{\mathcal{A}}=\frac{\operatorname{Area}\left(\gamma_{\mathcal{A}}\right)}{4 G_{N}}
$$

where $G_{N}$ is the Newton's constant in the bulk. While various aspects of different holographic superconductor models have been intensively studied (see, for example, Refs. [20, $21,22,23,24,25,26,27,28,30,31]$ ), the study of entanglement entropy in the holographic phase transition is just in the early stage. Ref. [32] studied the behavior of entanglement entropy in a holographic s-wave superconductor model, while Ref. [33] discussed the case in the holographic insulator/superconductor phase transition.

Note that a holographic p-wave superconductor (superfluid) with fully back reaction in the Einstein-Yang-Mills theory was constructed in Ref. [34]. This model is interesting not only because it is a holographic model to describe a p-wave superconducting phase transition, but also it contains a rich phase structure. There is a parameter $\alpha$, the ratio of the gravitational constant to the Yang-Mills coupling, in this model. The p-wave superconductor phase transition is second order for small $\alpha$, while it will become first order as $\alpha$ increases beyond a critical value. Therefore it is quite interesting to see the behavior of entanglement entropy in this model, in particular, to see how the entanglement entropy changes when the order of the phase transition changes.

The aim of this paper is just to investigate the behavior of entanglement entropy in the holographic p-wave superconductor at finite temperature. The entanglement entropy is calculated for a straight strip geometry at AdS boundary by using of the holographic 
proposal (1). We find that the behavior of entanglement entropy changes dramatically when the order of the phase transition changes. When the strip width is very large, i.e., $\gamma_{\mathcal{A}}$ probes deeply, the entanglement entropy is extensive as the thermal entropy of the bulk black hole, while in the opposite limit, the behavior perfectly fits the general form obtained from four-dimensional conformal theories. For the case with an intermediate strip width, by comparing the entanglement entropy and the thermal entropy of the bulk black holes during the whole process of phase transition, we see that they show the same behavior. This is an interesting and nontrivial result. As a result it shows that the entanglement entropy is a good probe to the holographic phase transition and that its behavior can indicate the appearance as well as the order of phase transition.

The paper is organized as follows. In Section (2), we briefly review the holographic p-wave superconductor model and give the complete equations of motion to be solved. In Section (3), the fully back-reacted system is solved by shooting method and basic behaviors in equilibrium are described. In Section (4), we explore the behaviors of the entanglement entropy in the p-wave superconductor phase transition. The conclusion and discussions are included in Section (5).

\section{Gravity Background}

We begin with the Einstein-Yang-Mills theory in five-dimensional asymptotically AdS spacetime

$$
S=\int d^{5} x \sqrt{-g}\left[\frac{1}{2 \kappa^{2}}\left(\mathcal{R}+\frac{12}{L^{2}}\right)-\frac{1}{4 \hat{g}^{2}} F_{\mu \nu}^{a} F^{a \mu \nu}\right],
$$

where $\kappa$ is the five dimensional gravitational constant connected with $G_{N}$ by the relation $2 \kappa^{2}=16 \pi G_{N}, \hat{g}$ is the Yang-Mills coupling constant and $L$ is the AdS radius. The $\mathrm{SU}(2)$ Yang-Mills field strength is

$$
F_{\mu \nu}^{a}=\partial_{\mu} A_{\nu}^{a}-\partial_{\nu} A_{\mu}^{a}+\epsilon^{a b c} A_{\mu}^{b} A_{\nu}^{c},
$$

where $\mu, \nu=(t, r, x, y, z)$ denote the indices of spacetime and $a, b, c=(1,2,3)$ are the indices of the $\mathrm{SU}(2)$ group generators $\tau^{a}=\sigma^{a} / 2 i$ ( $\sigma^{a}$ are Pauli matrices). $\epsilon^{a b c}$ is the totally antisymmetric tensor with $\epsilon^{123}=+1$. The gauge field is given by $A=A_{\mu}^{a} \tau^{a} d x^{\mu}$. Here we define a parameter $\alpha \equiv \kappa / \hat{g}$ which measures the strength of the back reaction.

Following Refs.[14, 34], our ansatz for the metric and Yang-Mills field are chosen by

$$
\begin{gathered}
d s^{2}=-N(r) \sigma(r)^{2} d t^{2}+\frac{1}{N(r)} d r^{2}+r^{2} f(r)^{-4} d x^{2}+r^{2} f(r)^{2}\left(d y^{2}+d z^{2}\right), \\
A=\phi(r) \tau^{3} d t+w(r) \tau^{1} d x .
\end{gathered}
$$


The independent equations of motion in terms of the above ansatz are deduced as follows

$$
\begin{aligned}
f^{\prime \prime} & =-\frac{\alpha^{2} f^{5} w^{2} \phi^{2}}{3 r^{2} N^{2} \sigma^{2}}+\frac{\alpha^{2} f^{5} w^{\prime 2}}{3 r^{2}}-f^{\prime}\left(\frac{3}{r}-\frac{f^{\prime}}{f}+\frac{N^{\prime}}{N}+\frac{\sigma^{\prime}}{\sigma}\right) \\
\phi^{\prime \prime} & =\frac{f^{4} w^{2} \phi}{r^{2} N}-\phi^{\prime}\left(\frac{3}{r}-\frac{\sigma^{\prime}}{\sigma}\right) \\
w^{\prime \prime} & =-\frac{w \phi^{2}}{N^{2} \sigma^{2}}-w^{\prime}\left(\frac{1}{r}+\frac{4 f^{\prime}}{f}+\frac{N^{\prime}}{N}+\frac{\sigma^{\prime}}{\sigma}\right) \\
\sigma^{\prime} & =\frac{\alpha^{2} f^{4} w^{2} \phi^{2}}{3 r N^{2} \sigma}+\sigma\left(\frac{2 r f^{\prime 2}}{f^{2}}+\frac{\alpha^{2} f^{4} w^{\prime 2}}{3 r}\right) \\
m^{\prime} & =\frac{\alpha^{2} r^{3} \phi^{\prime 2}}{6 \sigma^{2}}+\frac{r^{2} N \sigma^{\prime}}{2 \sigma}
\end{aligned}
$$

where $m(r)=\frac{r^{4}}{2 L^{2}}-\frac{r^{2}}{2} N(r)$ and " ।" denotes the derivative with respect to $r$. The event horizon $r=r_{H}$ is determined by the condition $N\left(r_{H}\right)=0$, which gives that $m\left(r_{H}\right)=\frac{r_{H}^{4}}{2 L^{2}}$. We should demand $\phi\left(r_{H}\right)=0$ to have a finite form for gauge field at horizon. The asymptotical behavior of these fields near the horizon are

$$
\begin{aligned}
\phi & =\phi_{H}^{(1)}\left(1-\frac{r_{H}}{r}\right)+\phi_{H}^{(2)}\left(1-\frac{r_{H}}{r}\right)^{2}+\ldots \\
w & =w_{H}^{(0)}+w_{H}^{(1)}\left(1-\frac{r_{H}}{r}\right)+\ldots \\
m & =\frac{r_{H}^{4}}{2 L^{2}}+m_{H}^{(1)}\left(1-\frac{r_{H}}{r}\right)+\ldots \\
\sigma & =\sigma_{H}^{(0)}+\sigma_{H}^{(1)}\left(1-\frac{r_{H}}{r}\right)+\ldots \\
f & =f_{H}^{(0)}+f_{H}^{(1)}\left(1-\frac{r_{H}}{r}\right)+\ldots
\end{aligned}
$$

All coefficients in above expansions are constants and are related by the equations of motion (6). After substituting the expansion into (6), we find only five independent parameters, i.e., $\left\{r_{H}, \phi_{H}^{(1)}, w_{H}^{(0)}, \sigma_{H}^{(0)}, f_{H}^{(0)}\right\}$.

The ultraviolet (UV) asymptotic expansion near the boundary $r \rightarrow \infty$ behaves as

$$
\begin{gathered}
\phi=\phi_{B}^{(0)}+\frac{\phi_{B}^{(2)}}{r^{2}}+\ldots, \quad w=w_{B}^{(0)}+\frac{w_{B}^{(2)}}{r^{2}}+\ldots, \\
m=m_{B}^{(0)}+\frac{m_{B}^{(2)}}{r^{2}}+\ldots, \quad \sigma=\sigma_{B}^{(0)}+\frac{\sigma_{B}^{(4)}}{r^{4}}+\ldots, \quad f=f_{B}^{(0)}+\frac{f_{B}^{(4)}}{r^{4}}+\ldots
\end{gathered}
$$

To recover the pure AdS boundary, we need the boundary conditions $\sigma_{B}^{(0)}=1$ and $f_{B}^{(0)}=1$. $\phi_{B}^{(0)}$ is the chemical potential $\mu$ and $w_{B}^{(0)}$ is the source of the operator $\hat{J}_{1}^{x}$. To spontaneously break the $\mathrm{U}(1)$ gauge symmetry and rotational symmetry, we should impose $w_{B}^{(0)}=0$.

After imposing boundary conditions, the equations of motion can be solved numerically via tuning the four independent parameters $\left\{\phi_{H}^{(1)}, w_{H}^{(0)}, \sigma_{H}^{(0)}, f_{H}^{(0)}\right\}$ to search for solutions that 
meet the requirements $\sigma_{B}^{(0)}=1, f_{B}^{(0)}=1, w_{B}^{(0)}=0$. Notice that the above equations of motion (6) have four useful scaling symmetries [34]

$$
\begin{gathered}
\sigma \rightarrow \lambda \sigma, \quad \phi \rightarrow \lambda \phi, \\
f \rightarrow \lambda f, \quad \omega \rightarrow \lambda^{-2} \omega, \\
r \rightarrow \lambda r, \quad m \rightarrow \lambda^{2} m, \quad L \rightarrow \lambda L, \quad \phi \rightarrow \lambda^{-1} \phi, \quad \alpha \rightarrow \lambda \alpha, \\
r \rightarrow \lambda r, \quad\{t, x, y, z\} \rightarrow \lambda^{-1}\{t, x, y, z\}, \quad m \rightarrow \lambda^{4} m, \quad \omega \rightarrow \lambda \omega, \quad \phi \rightarrow \lambda \phi .
\end{gathered}
$$

Taking advantage of the scaling symmetries (9) and (10), we will first choose $\sigma_{H}^{(0)}=1, f_{H}^{(0)}=$ 1 in our shooting method, then use the two scaling symmetries again to set $\sigma_{B}^{(0)}=1, f_{B}^{(0)}=$ 1. The last two scaling symmetries allow us to set $L=r_{H}=1$.

\section{Thermodynamics and Phase Transition}

From the discussion in Section 2, for given $\left\{\phi_{H}^{(1)}, w_{H}^{(0)}, \alpha\right\}$, we can solve the equations of motion (6) by choosing $\phi_{H}^{(1)}$ as a shooting parameter. After solving the coupled equations, we can obtain the condensate $\left\langle\hat{J}_{1}^{x}\right\rangle=\frac{2 \alpha^{2}}{\kappa^{2}} w_{B}^{(2)}$, chemical potential $\mu$ and total charge density $\rho=\frac{2 \alpha^{2}}{\kappa^{2}} \phi_{B}^{(2)}$ by just reading off the coefficients $w_{B}^{(2)}, \phi_{B}^{(0)}$ and $\phi_{B}^{(2)}$ from (8) respectively. However, there is an analytic black hole solution of (6) for vanishing $\omega(r)$, which is just the RN-AdS black hole

$$
\begin{gathered}
\phi(r)=\mu\left(1-\frac{r_{H}^{2}}{r^{2}}\right), \quad \omega(r)=0, \quad \sigma(r)=f(r)=1, \\
N(r)=-\frac{2}{r^{2}}\left(-\frac{\alpha^{2} \mu^{2} r_{H}^{4}}{3} \frac{1}{r^{2}}+\frac{\alpha^{2} \mu^{2}}{3} r_{H}^{2}+\frac{1}{2} r_{H}^{4}\right)+r^{2}
\end{gathered}
$$

This so called RN-AdS solution has vanishing $\omega(r)$, thus corresponds to the normal phase. From AdS/CFT correspondence, the boundary thermal equilibrium states are dual to black hole geometries in bulk, and the Hawking temperature of black hole is considered as the temperature of the boundary theory [35]. From the metric ansatz (4), the Hawking temperature of the black hole is

$$
T=\left.\frac{\sigma N^{\prime}}{4 \pi}\right|_{r=r_{H}}=\left.\left(\frac{\sigma}{\pi L^{2}}-\alpha^{2} \frac{\phi^{\prime 2}}{12 \pi \sigma}\right) r\right|_{r=r_{H}} .
$$

The Bekenstein-Hawking entropy of this black hole is

$$
S_{T}=\frac{A_{H}}{4 G_{N}}=\frac{2 \pi}{\kappa^{2}} V r_{H}^{3},
$$

where $A_{H}$ denotes the area of the horizon and $V=\int d x d y d z$. 
We will work in the grand canonical ensemble where the chemical potential $\mu$ at the boundary is fixed. It is convenient to express physical quantities in scale invariant way. Under the scaling symmetry (12), the relevant quantities scale as follows

$$
\mu \rightarrow \lambda \mu, \quad T \rightarrow \lambda T, \quad \rho \rightarrow \lambda^{3} \rho, \quad\left\langle\hat{J}_{1}^{x}\right\rangle \rightarrow \lambda^{3}\left\langle\hat{J}_{1}^{x}\right\rangle .
$$

Therefore, we choose the following scale invariant combinations to examine physics in the grand canonical ensemble

$$
\frac{T}{\mu}, \frac{\rho}{\mu^{3}}, \frac{\left\langle\hat{J}_{1}^{x}\right\rangle}{\mu^{3}} .
$$

In gauge/gravity duality the grand potential $\Omega$ of the boundary thermal state is identi-
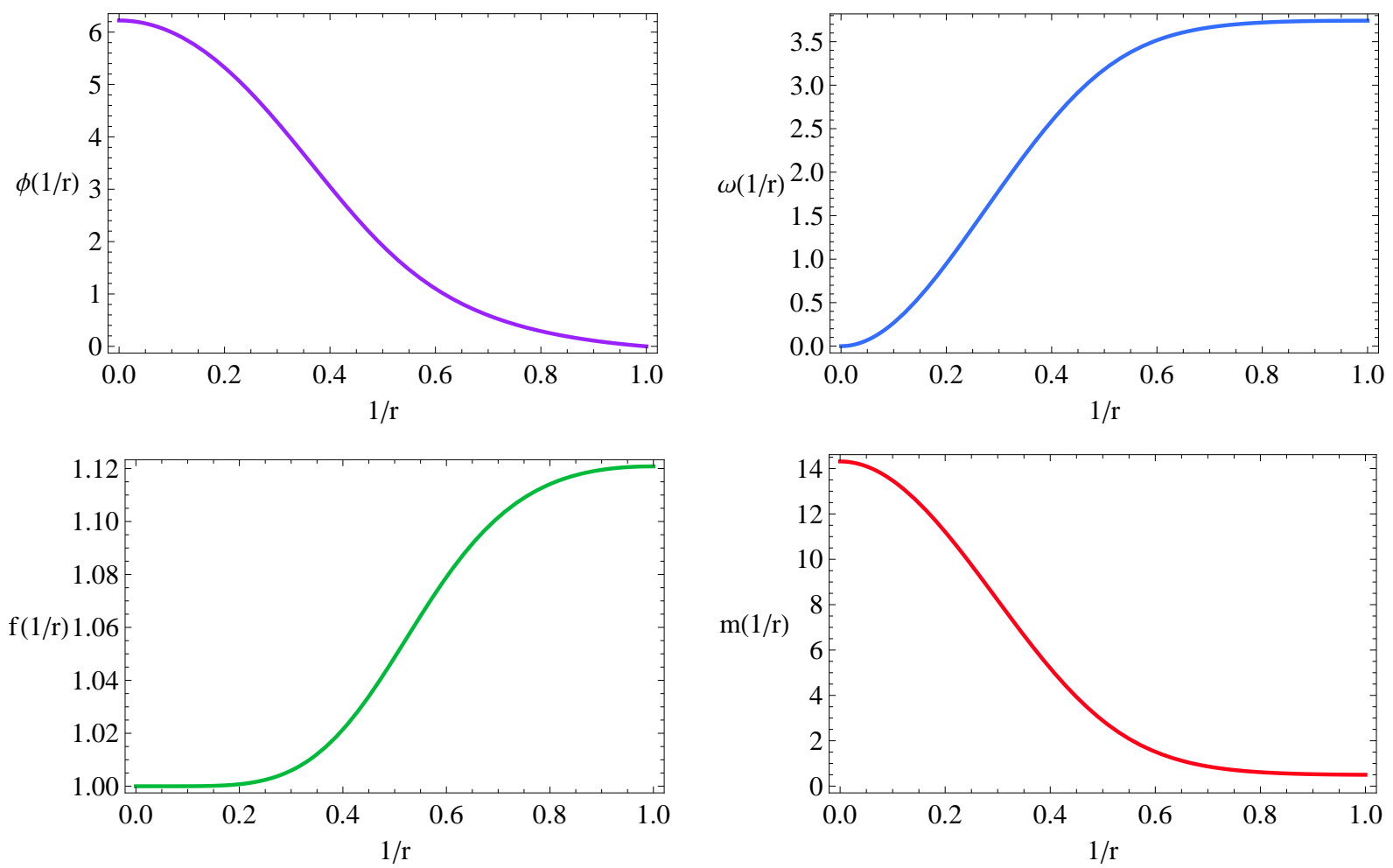

Figure 1: The configurations of gauge fields $(\phi, \omega)$ and metric functions $(f, m)$ as a function of the inverse holographic coordinate $1 / r$ for $\alpha=0.447$ at $T / \mu \simeq 0.0215$.

fied with $T$ times the on-shell bulk action in Euclidean signature. The Euclidean action must include the Gibbons-Hawking boundary term for a well-defined Dirichlet variational principle and further a surface counterterm for removing divergence

$$
S_{\text {Euclidean }}=-\int d^{5} x \sqrt{g}\left[\frac{1}{2 \kappa^{2}}\left(R+\frac{12}{L^{2}}\right)-\frac{1}{4 \hat{g}^{2}} F_{\mu \nu}^{a} F^{a \mu \nu}\right]+\frac{1}{2 \kappa^{2}} \int_{r \rightarrow \infty} d^{4} x \sqrt{h}\left(-2 K+\frac{6}{L^{2}}\right),
$$

where $h$ is the induced metric on the boundary $r \rightarrow \infty$, and $K$ is the trace of the extrinsic curvature. 
This model has been numerically solved in Ref. [34] and shown that the order of the phase transition relies on the value of $\alpha$. The transition is second order as $\alpha$ is less than $\alpha_{c}=0.365 \pm 0.001$, while it is first order for larger values than $\alpha_{c}$. We will re-solve the equations of motion (6) for completeness and for further discussion. Typical solutions for the metric and gauge field configurations are presented in Figure.(1), which are needed to calculate entanglement entropy in the next section.

To compare the differences between the second order transition and first order transition, we choose $\alpha=0.316<\alpha_{c}$ and $\alpha=0.447>\alpha_{c}$ as concrete examples. The conden-

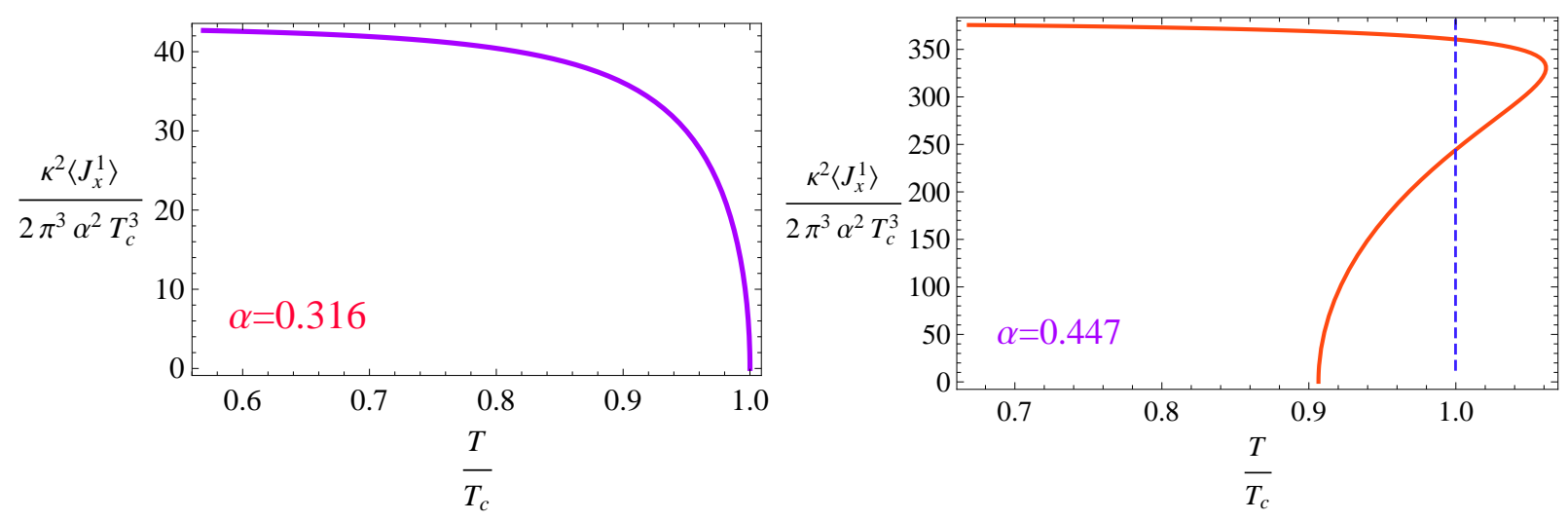

Figure 2: The condensation of vector operator $\hat{J}_{1}^{x}$ as a function of temperature for $\alpha=0.316$ (left plot) and $\alpha=0.447$ (right plot). The vertical dashed line on the right plot represents the transition temperature. The right plot has a region where $\left\langle\hat{J}_{1}^{x}\right\rangle$ is multi-valued.

sation of vector operator $\hat{J}_{1}^{x}$ as a function of temperature for $\alpha=0.316$ and $\alpha=0.447$ is displayed in Figure.(2). For the case $\alpha=0.316$, the condensate $\left\langle\hat{J}_{1}^{x}\right\rangle$ appears at a particular temperature $T_{c} \simeq 0.0458 \mu$. As the temperature is lowered, $\left\langle\hat{J}_{1}^{x}\right\rangle$ increases continuously. The critical behavior near $T_{c}$ is found to be $\left\langle\hat{J}_{1}^{x}\right\rangle \propto\left(1-T / T_{c}\right)^{\frac{1}{2}}$, which is the typical result from the mean-field theory. While for the case $\alpha=0.447$, we can see from Figure.(2) that the curve of the condensate has two branches when $0.905<T / T_{c}<1.06$. Therefore, the value of condensate has a jump at critical temperature $T_{c} \simeq 0.0218 \mu$, which represents a first order phase transition.

To distinguish which branch is physical, i.e., thermodynamically favored, we need to calculate the grand potential $\Omega$. The values of grand potential $\Omega$ are exhibited in Figure.(3). The RN-AdS solutions always exist for all temperatures, but it is only thermodynamically favored at high temperatures $T>T_{c}$. As the temperature is lowered below critical value for each $\alpha$, the grand potential $\Omega$ from superconductor solution is smaller than the one from the RN-AdS solution, thus the superconductor phase is physically compared to the normal phase (RN-AdS solution). Therefore, there is a phase transition occurring at $T_{c}$. For $\alpha=0.316$ case, the transition is second order. However, for $\alpha=0.447$ case, there is a characteristic "swallowtail" shape of the grand potential, signaling a first order transition.

According to the two-fluid model, the total charge density $\rho$ can be divided into two components $\rho=\rho_{n}+\rho_{s}$, where $\rho_{n}$ is the normal component, while $\rho_{s}$ is the superconducting 

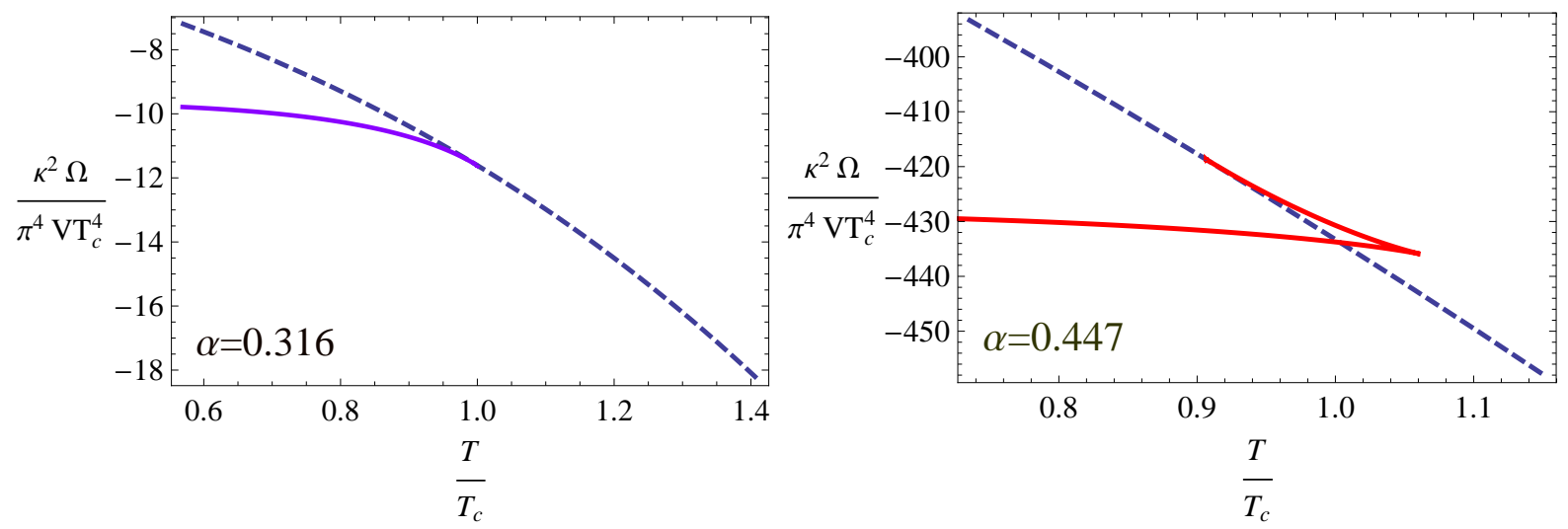

Figure 3: The grand potential $\Omega$ as a function of temperature for $\alpha=0.316$ (left plot) and $\alpha=0.447$ (right plot). Trace the physical curve by choosing the lowest grand potential at a fixed $T$. The critical temperature $T_{c}$ is the point at which the superconductor phase begins to be thermodynamically preferred. In both plots, dashed blue curves are for the RN-AdS solutions, while the solid curves are for the superconductor solutions.

component. In the holographic setup, the normal charge density $\rho_{n}$ is proportional to the $\tau^{3}$ part of the electric field at the horizon [14], which is given by $\frac{\alpha^{2}}{\kappa^{2}} \phi_{H}^{(1)}$ in our units. Therefore the superconducting charge density is $\rho_{s}=\rho-\rho_{n}$, where $\rho=\frac{2 \alpha^{2}}{\kappa^{2}} \phi_{B}^{(2)}$ is the total charge density. We draw $\frac{\rho_{s}}{\rho}$ and $\frac{\rho_{n}}{\rho}$ versus temperature in Figure.(4). $\frac{\rho_{s}}{\rho}$ and $\frac{\rho_{n}}{\rho}$ as two functions of temperature are reminiscent of the temperature dependence of the superfluid and normal components of liquid He II as measured from in the torsional oscillation disk stack experiment. $\rho_{s}$ seems to vanish near $T_{c}$ like a power law, i.e., $\left(T_{c}-T\right)^{\nu}$. However, we find $\frac{\rho_{s}}{\rho}$ goes to zero linearly here, while the experiment gives a value of $\nu \simeq 0.67$.

\section{Entanglement Entropy}

After solving the equations of motion, we are now ready to calculate the entanglement entropy in this holographic model. Because of the arbitrary choice of the subsystem $\mathcal{A}$, we can define infinite entanglement entropies correspondingly. However, we are here interested in a belt geometry with a finite width $\ell$ along the $x$ direction and infinitely extending in $y$ and $z$ directions.

To deal with the UV divergence, we assume that the subsystem $\mathcal{A}$ sites on the slice $r=\frac{1}{\epsilon}$ where $\epsilon \rightarrow 0$ is the UV cutoff. More specifically, $\gamma_{A}$ starts from $x=-\frac{\ell}{2}$ at $r=\frac{1}{\epsilon}$, extends into the bulk until it reaches the minimum $r=r_{*}$, then returns back to the AdS boundary $r=\frac{1}{\epsilon}$ at $x=+\frac{\ell}{2}$. According to the proposal (1), we need to minimize the following area functional

$$
\operatorname{Area}\left(\gamma_{\mathcal{A}}\right)=V_{2} \int_{-\frac{\ell}{2}}^{+\frac{\ell}{2}} d x \sqrt{\frac{r^{4} f(r)^{4}}{N(r)}\left(\frac{d r}{d x}\right)^{2}+r^{6}}
$$




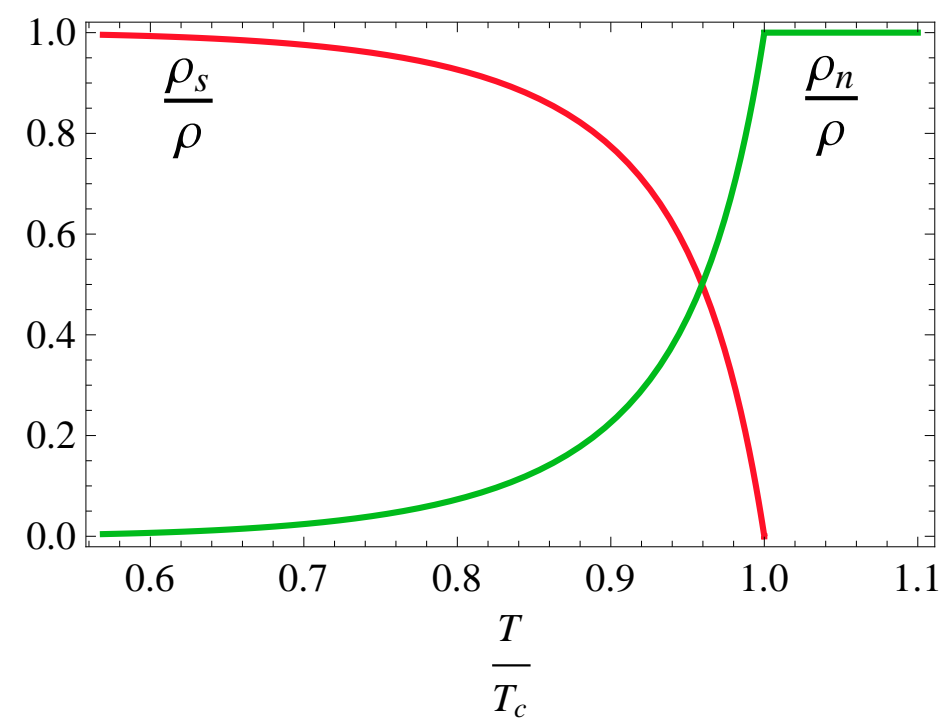

Figure 4: $\frac{\rho_{s}}{\rho}$ and $\frac{\rho_{n}}{\rho}$ are plotted versus temperature in $\alpha=0.316$ case. In our numerical calculation, we find $\frac{\rho_{s}}{\rho}$ goes to zero linearly near $T_{c}$, which is the same as the critical behavior obtained in the probe limit.

where $V_{2}=\int d y d z$. The integrand can be considered as the Lagrangian with $x$ direction thought of as time. As the Lagrangian does not explicitly depend on "time $x$ ", the Hamiltonian is conserved. Thus we can easily deduce the "equation of motion" that gives minimal area from (19)

$$
\frac{d r}{d x}= \pm \frac{r \sqrt{N(r)}}{f(r)^{2}} \sqrt{\frac{r^{6}}{r_{*}^{6}}-1}
$$

where we demand that the surface is smooth at the turning point $r=r_{*}$, i.e., $d r /\left.d x\right|_{r=r_{*}}=$ 0 . Integrated once, the belt width $\ell$ can be fixed as

$$
\frac{\ell}{2}=\int_{r_{*}}^{\frac{1}{\epsilon}} d r \frac{d x}{d r}=\int_{r_{*}}^{\frac{1}{\epsilon}} d r \frac{f(r)^{2}}{r \sqrt{N(r)}} \frac{r_{*}^{3}}{\sqrt{r^{6}-r_{*}^{6}}} .
$$

Substituting (20) into (19), we finally obtain the entanglement entropy

$$
S_{E}=\frac{V_{2}}{2 G_{N}} \int_{r_{*}}^{\frac{1}{\epsilon}} d r \frac{r^{5} f(r)^{2}}{\sqrt{N(r)}} \frac{1}{\sqrt{r^{6}-r_{*}^{6}}}=\frac{2 \pi}{\kappa^{2}} V_{2}\left(\frac{1}{\epsilon^{2}}+S_{E}\right),
$$

where the UV cutoff $1 / \epsilon$ has been taken into consideration. The first term indicates UV divergent $(\epsilon \rightarrow 0)$ and represents the "area law" [17,36]. It can be deduced by plugging the UV asymptotic expansion (8) into (22). While the second term is independent of the cutoff and is finite, so this term is physical important. Following the discussion in Section (3), $\ell$ and $S_{E}$ under the transformation (12) scale as $\ell \rightarrow \lambda^{-1} \ell, \quad S_{E} \rightarrow \lambda^{2} S_{E}$, so we will introduce the scale invariants

$$
\mu \ell, \frac{S_{E}}{\mu^{2}} .
$$




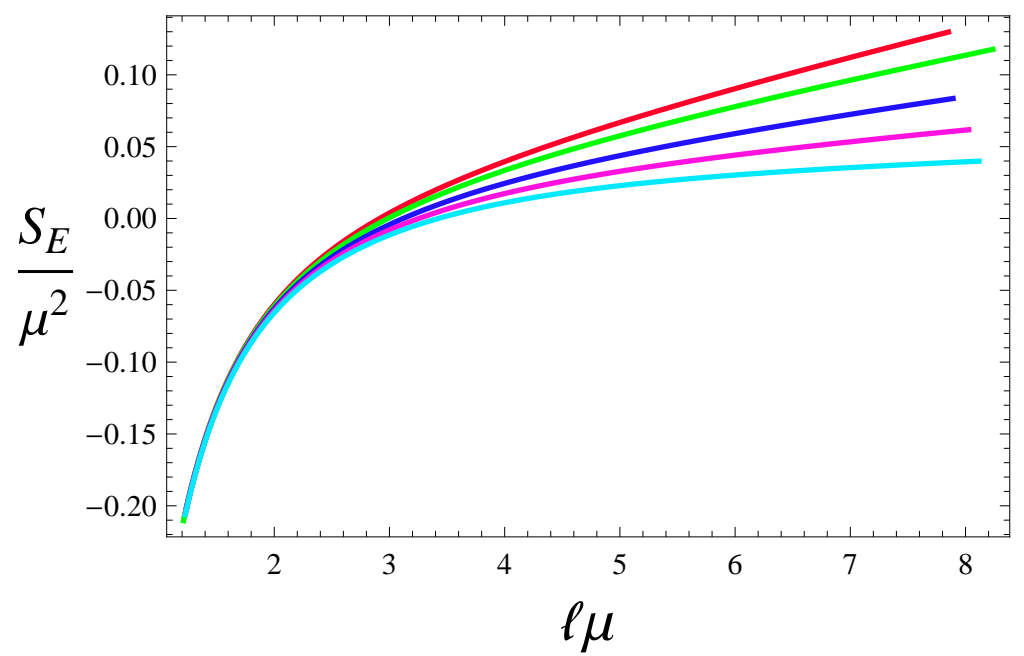

Figure 5: The entanglement entropy in $\alpha=0.316$ case, as a function of belt width at fixed temperature. The curves from top to bottom correspond to $T \simeq$ $0.0458 \mu, 0.0448 \mu, 0.0424 \mu, 0.0387 \mu$, and $0.0294 \mu$, respectively.

We first focus on the case with second order phase transition, i.e., $\alpha<\alpha_{c}$. In Figure(5) we plot the behavior of the universal part of the entanglement entropy $S_{E}$ as a function of belt width $\ell$ by fixing the temperature. The curve at the top is at the transition temperature $T_{c}$, which is identical with the RN-AdS case. We observe that the slope of the curve decreases as the temperature is lowered in superconductor situation as is expected that the lower the temperature is, the more the degrees of freedom will condense. This phenomenon can be seen much more clearly in Figure(6), which shows how the entanglement entropy evolves with temperature by fixing the belt width. We see that although the entanglement entropy is continuous at critical temperature $T_{c}$, there is a discontinuous change in its slope at $T_{c}$. This discontinuity may signal a significant reorganization of the degrees of freedom of the system, since some kind of new degrees of freedom, like the Cooper pair, would emerge in the new phase.

We show the results for the first order transition case in Figure(7). The behavior of entanglement entropy at fixed temperature is quite similar to the second order transition case. $S_{E}$ changes monotonously with respect to the belt width. This behavior is quite different from the result in Ref. [32], where a swallowtail shape appears in a region with finite belt width. It can be seen from the left plot in Figure(7) that the belt width monotonously decreases as the turning point $r_{*}$ increases. Actually, $\ell$ diverges logarithmically as $r_{*} \rightarrow r_{H}$ at nonvanishing temperature as observed from (21). In fact in Ref. [32], the non-monotonousness of $\ell$ with respect to the turning point $r_{*}$ is indispensable for the emergence of the kink. On this we will have more discussions in the last section of the paper.

The behavior of $S_{E}$ as a function of temperature for fixed belt width is presented in Figure(8). Comparing with Figure(6), we can find a dramatic change in the $\alpha>\alpha_{c}$ 

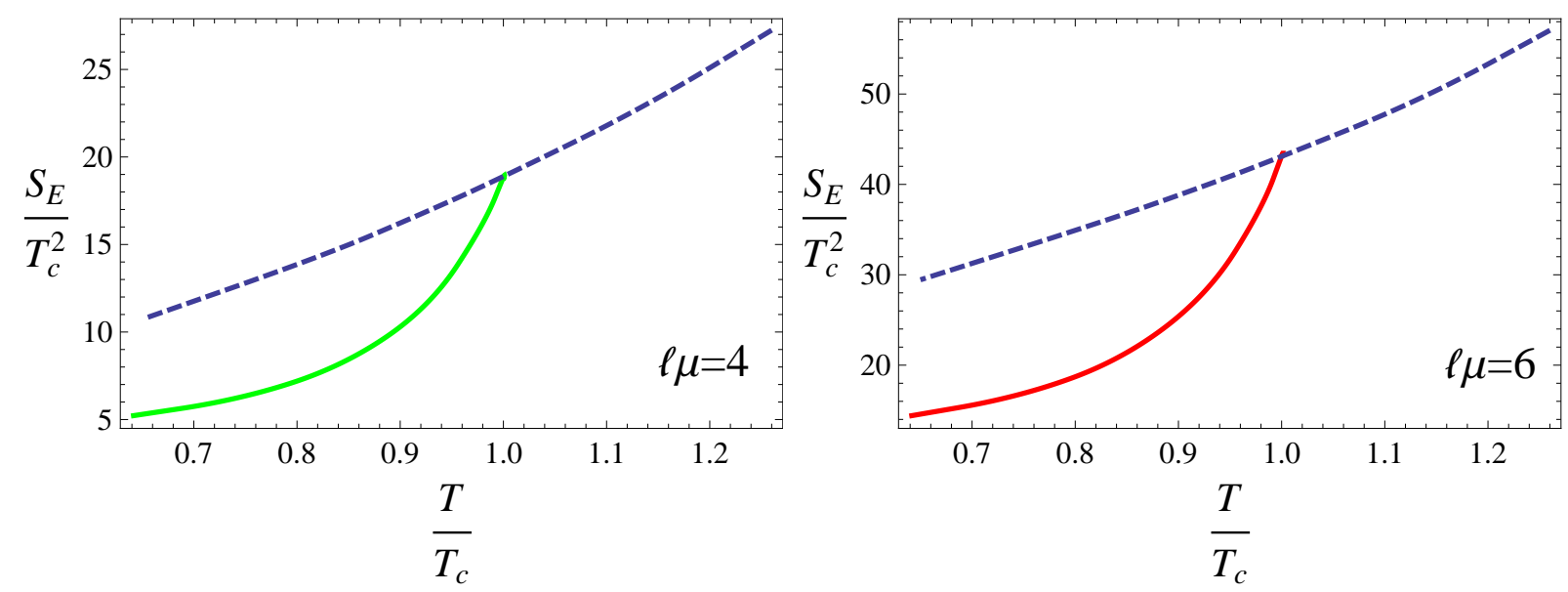

Figure 6: The entanglement entropy in $\alpha=0.316$ case, as a function of temperature at fixed belt width (left plot for $\ell \mu=4$ and right plot for $\ell \mu=6$ ). The dashed blue curves are from the RN-AdS solutions, while the solid curves are from superconductor solutions. The physical curve is determined by selecting the lower entropy at a given $T$.

case. The blue curve from normal phase is physical as $T>T_{c}$, while the curve with the lowest entropy at a given temperature is preferred below $T_{c}$. Therefore, there is an obvious jump in $S_{E}$ as well as its slope at critical temperature. It seems reasonable to expect an abrupt reduction in the number of degrees of freedom at $T_{c}$ since the condensate has a sharp jump at the critical point.

In both two kinds of phase transition, we observe from Figure.(5) and Figure.(7) that $S_{E}$ exhibits linear behavior with respect to $\ell$ for large $\ell$. Indeed, we can find that in large $\ell \mu \sim \ell T$ limit, the main contribution of the integrals (21) and (22) to $S_{E}$ comes from the region near $r=r_{*} \sim r_{H}$. In addition to $N(r) \propto\left(r-r_{H}\right)$ near horizon at nonvanishing temperature, we can deduce the linear relation $\frac{S_{E}}{\mu^{2}} \sim \ell \mu$. Thus we obtain the entanglement entropy in this limit

$$
S_{E} \sim \frac{2 \pi}{\kappa^{2}} \mu^{3} V_{2} \ell \sim \frac{2 \pi}{\kappa^{2}} T^{3} V
$$

where we have subtracted the UV divergent term. This equation is similar to the BekensteinHawking entropy (15) and may seem to be surprised at first glance since the entropy (24) is proportional to the area of the subsystem $\mathcal{A}$ as opposed to the area law (1). A geometric interpretation made in Ref. [17] is that $\gamma_{\mathcal{A}}$ will wrap a part of the black hole horizon as the increase of belt width and therefore is equal to the fraction of black hole entropy .

In contrast, from Figure.(5), and Figure.(7), the value of $\frac{S_{E}}{\mu^{2}}$ seems to be power-law divergent as $\mu \ell$ vanishes. In fact, the relationship between entanglement entropy and belt width in small $\ell \mu \sim \ell T$ limit is found to perfectly agree with a universal function in our numerical calculation

$$
S_{E}=\frac{2 \pi}{\kappa^{2}} V_{2}\left(\frac{1}{\epsilon^{2}}-\frac{0.32066}{\ell^{2}}\right)=\frac{2 \pi}{\kappa^{2}} T^{2} V_{2}\left(\frac{1}{\epsilon^{2} T^{2}}-\frac{0.32066}{\ell^{2} T^{2}}\right) .
$$



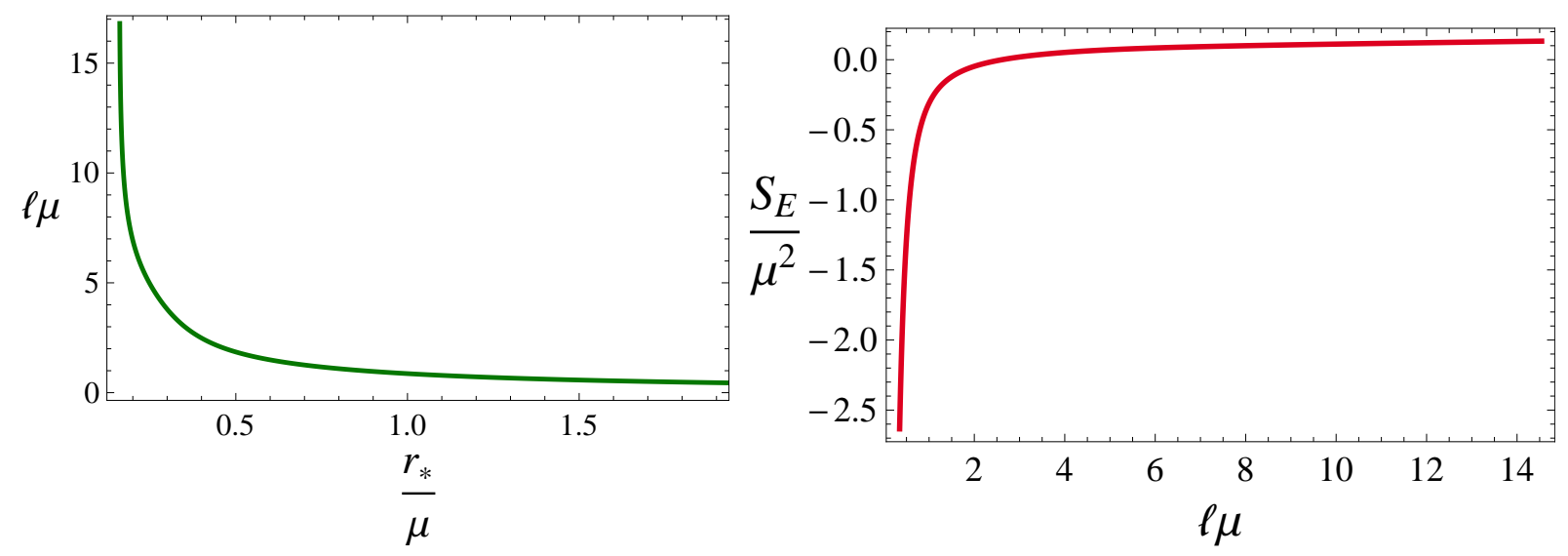

Figure 7: The left plot shows the behavior of belt width as a function of turning point $r_{*}$ for $\alpha=0.447$ at $T / \mu \simeq 0.0215$. While the entanglement entropy as a function of belt width is presented in the right plot.

Notice that entanglement entropy in four-dimensional conformal theories for a belt configuration takes the universal form $[37,38]$

$$
S_{\mathcal{A}}=\zeta \frac{V_{2}}{\epsilon^{2}}-C \frac{V_{2}}{2 \ell^{2}}
$$

where $\zeta$ and $C$ are numerical constants which depend on the details of a theory under consideration. Our $\ell T \rightarrow 0$ result (25) has the same form as (26), which is reasonable since our metric solution is asymptotically $\mathrm{AdS}$ and $\gamma_{\mathcal{A}}$ with small belt length can only probe the bulk sufficiently near the boundary $r \rightarrow \infty$. As discussed in [39], there exist some universal crossover functions connecting the universal parts of the entanglement entropy to the thermal entropy. We try to construct the crossover function here as $S_{E}(\ell, T)=$ $T^{n} F(\ell T)$. In our situation, as $\ell T \rightarrow \infty$, we obtain that $\mathrm{n}=2$ and $F(\xi \rightarrow \infty) \sim \xi$, thus $S_{E}(\ell, T)$ behaves as the extensive thermal entropy. While as $\ell T \rightarrow 0$, we find $n=2$ and $F(\xi \rightarrow 0) \sim \frac{1}{\xi^{2}}$. For the intermediate scale, the precise form of $F(\xi)$ can only be obtained numerically.

To get further understanding of the connection between the entanglement entropy and thermal entropy, it would be irradiative and instructive to compare the behaviors of them during the process of phase transition. Figure.(9) shows the thermal entropy $S_{T}$ as a function of temperature. The quite qualitatively similarity in Figure.(6), Figure.(8) and Figure.(9) is impressive and striking. Note that $\ell \mu=4$ and $\ell \mu=6$ in Figure(6) and Figure(8), this result is nontrivial since the belt width here is neither too large nor too small. The minimal surface $\gamma_{\mathcal{A}}$ will not be very close to the horizon. Due to the lake of numerical control at very low temperature, compared to the critical temperature of the phase transition, we can not plot all points in superconducting phase (the lowest temperature in Figure(6) and Figure(8) is about $0.64 T_{c}$ and $0.68 T_{c}$ separately). However, it is not obtrusive to conclude that the entanglement entropy does behave as thermal entropy at least for not very low temperature. Furthermore, as the thermal entropy is equivalent to the black hole en- 

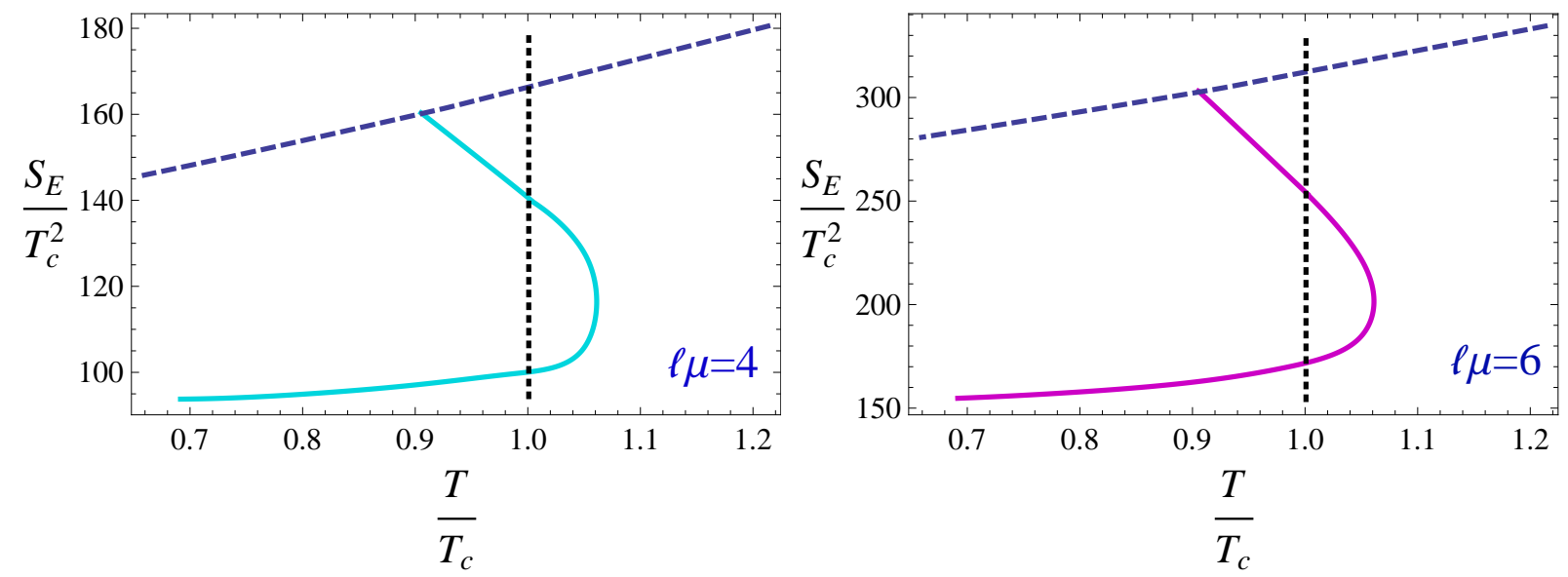

Figure 8: The entanglement entropy in $\alpha=0.447$ case, as a function of temperature at fixed belt width (left plot for $\ell \mu=4$ and right plot for $\ell \mu=6$ ). The dashed blue curves are from the RN-AdS solutions, while the solid curves are from the superconductor solutions. The physical curve is determined by choosing the dashed blue curve above $T_{c}$, indicated by the vertical dotted line, and the curve which has the lowest entropy below $T_{c}$.

tropy in the holographic setup, our calculation seems to support the viewpoint that black hole entropy is due to the entanglement entropy [40, 41, 42, 43].

\section{Conclusion and discussions}

In a recent paper [33], we reported the behavior of entanglement entropy in the holographical insulator/superconductor phase transition, where a non-monotonic behavior of the entanglement entropy was found as the change of chemical potential. Because of the absence of horizon in the soliton background, both the temperature and thermal entropy in dual boundary system do vanish. It is unable to extract the relationship between entanglement entropy and thermal entropy or black hole entropy. In this paper we overcome the shortcoming by studying the holographic p-wave superconductor at finite temperature. The other motivation is to see how the behavior of the entanglement entropy will change as we tune the parameter $\alpha$, the ratio of the gravitational constant to the Yang-Mills coupling constant, which can change the order of the phase transition.

In the fully back reacted case, we found $\frac{\rho_{s}}{\rho}$ scales as $T-T_{c}$ near critical point, which is the same as the result in the probe limit [14]. As noted in Ref. [14] this scaling behavior is different from the transition between superconductivity and pseudogap state of high $T_{c}$ materials, where the fraction is finite and nonvanishing. The behavior is reminiscent of superfluid properties of He II. However, the critical exponent in He II is about 0.67, while here it is one.

We found that the qualitative behavior of entanglement entropy is dramatically different for sufficiently small and large $\alpha$ cases as we lower the temperature. For the case $\alpha<\alpha_{c}$, 

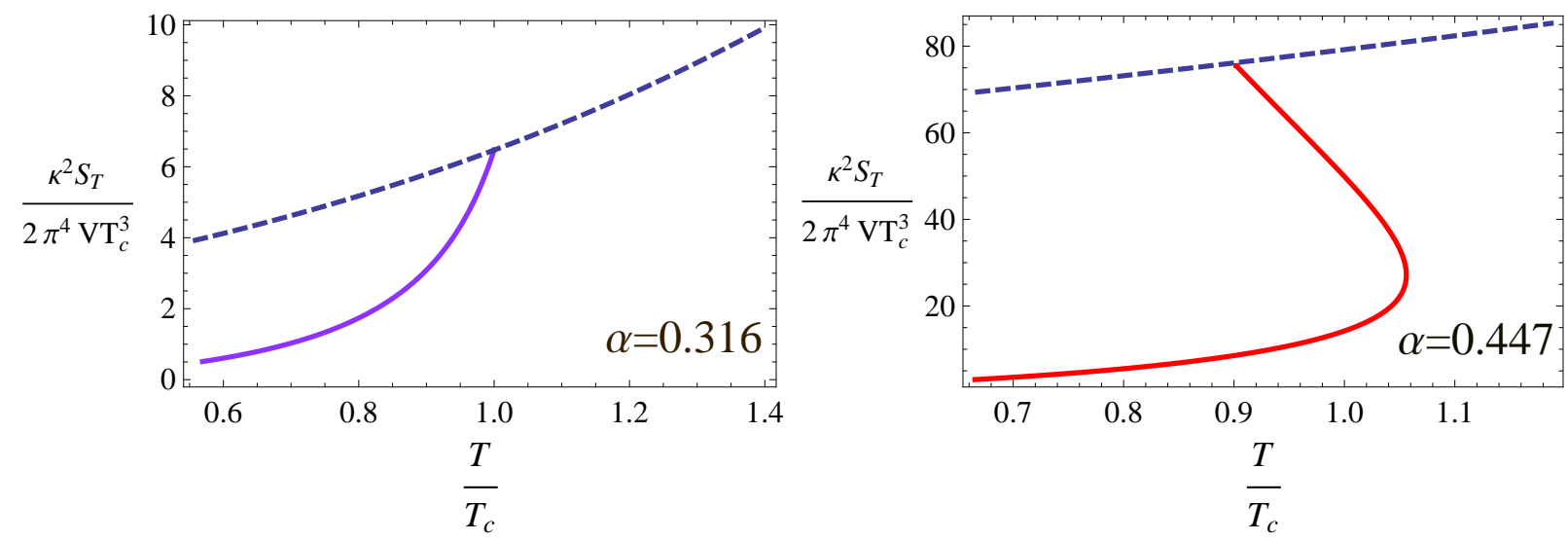

Figure 9: The thermal entropy $S_{T}$ as a function of temperature for $\alpha=0.316$ (left plot) and $\alpha=0.447$ (right plot). The dashed blue curves are from the RN-AdS solutions, while the solid curves are from the superconductor solutions. A discontinuous slope at $T_{c}$ in the left plot obviously indicates a second-order transition, while the jump in the right plot indicates a first-order transition.

the entanglement entropy is continuous at the critical temperature $T_{c}$, while it has a jump for $\alpha>\alpha_{c}$ case. That is, the behavior of the entanglement entropy shows that the phase transition is second order when $\alpha<\alpha_{c}$, while it is first order as $\alpha>\alpha_{c}$.

When the belt width is very large, i.e., $\gamma_{\mathcal{A}}$ probes deeply into the bulk, the entanglement entropy is extensive as the thermal entropy of the bulk black holes. In the opposite limit, the behavior perfectly fits the general form obtained from four-dimensional conformal theories. Motivated by Ref. [39], there may exists a crossover function connecting the two limits. We compared the behaviors of the entanglement entropy and thermal entropy during the whole process of the superconductor phase transition. It shows that they behave qualitatively the same. This result is nontrivial since the belt width here is neither too large nor too small (note that $\ell \mu=4$ and $\ell \mu=6$ in Figure.(6) and Figure.(8)). From the minimal surface picture, the minimal surface $\gamma_{\mathcal{A}}$ can not be very close to "hugging" the horizon at these intermediate scales. Due to the lack of numerical control at low temperature, in our numerical calculation we are not able to display the behavior of the entanglement entropy at sufficiently low temperature, compared to the critical temperature of the phase transition. As a result, we can conclude that the entanglement entropy is a good probe to the holographic pase transition, and that its behavior can indicate not only the appearance, but also the order of the phase transition.

Here it is quite interesting to compare our results with those from the case with holographic s-wave superconductors in Ref. [32]. The model studied there is a $S O(3) \times$ $S O$ (3) invariant truncation of four-dimensional $\mathcal{N}=8$ gauged supergravity [44]. Different from other "top-down" models of holographic superconductors (see, for example, Refs. [45, 46, 47]), where the superconductor phase transition is a second order one, the phase transition studied in Ref. [32] will be second order or first order, depending on the boundary condition of a scalar field in the model. In the second order phase transition 
case, the entanglement entropy shows the feature of the second order phase transition: the entanglement entropy is continuous and its slop has a jump at the critical temperature. On the order hand, in the case of first order phase transition, there exists not only a jump for the entanglement entropy at the critical temperature, but also a kink shape (swallowtail shape) for the entanglement entropy for a given range of strip widths in the superconducting phase. The kink persists even for zero temperature solutions. It was argued there that the appearance of the kink can be attributed to the existence of a new scale in the theory and the entanglement entropy is a good probe of the new scale. The new scale can be viewed as a finite correlation length. Quite interestingly, in our p-wave superconductor model, the kind does not appear even in the first order phase transition case (see Figure.7). This suggests that the existence of this new scale is not universal in holographic superconductor models. Note that in this p-wave model, not only the $U(1)$ symmetry but also the rotational symmetry are broken, hence, the superconducting phase is anisotropic. On the other hand, only the $U(1)$ symmetry is broken in the s-wave model studied in Ref. [32] and the superconducting phase is isotropic. We suspect that the anisotropy might be responsible for the absence of the kink in the superconducting phase in the first order transition case. Clearly it would be quite interesting to investigate whether such kink is common in other s-wave superconductor models.

Note that the zero temperature solution studied in Ref. [32] is an RG flow between two AdS spaces. Indeed, the existence of the kink for the entanglement entropy in such backgrounds has also been observed in Ref. [48] (see also Ref. [49]) where null energy condition is used to constrain the monotonic behavior of c-function along RG flows. It was shown there that the kink would emerge for particular choice of the asymptotically AdS geometry, which is obviously shown in Figure(6) in Ref. [48]. More specifically, the geometry is controlled by a parameter $R$. A kink will emerge for $R<R_{c}$, while it will disappear for the case $R>R_{c}$. In Ref. [32], it has been also analyzed in some details when the kink will appear. It shows that the presence of the kink is due to the particular potential in the model which in turn further determines the geometry structure of the background solutions. Let us further stress here that the kink of entanglement entropy also appears in the AdS soliton backgrounds $[38,50,33]$, and the signal of the kink acts as the "confinement/deconfiment" phase transition.

Finally we would like to mention that the holographic p-wave superconductor model has been extended to include the Gauss-Bonnet term in Refs. [51, 52] where the condensate is found to become harder as the Gauss-Bonnet coefficient grows up. On the other hand, the original entanglement entropy proposal has been generalized to include some higher derivative corrections, such as the Gauss-Bonnet term, in the bulk [53, 54]. The entanglement entropy in the Gauss-Bonnet gravity has been calculated for many configurations and discovered to give additional contributions $[48,55,56]$. There might be some new features for the entanglement entropy in the p-wave superconductor models with the Gauss-Bonnet term. We wish to report on the related work in future. 


\section{Acknowledgements}

We would like to thank Hai-Qing Zhang, Zhang-Yu Nie, and Shu-Hao Zou for their helpful discussions and suggestions. This work was supported in part by the National Natural Science Foundation of China (No.10821504, No.10975168 and No.11035008), and in part by the Ministry of Science and Technology of China under Grant No. 2010CB833004.

\section{References}

[1] J. M. Maldacena, "The large N limit of superconformal field theories and supergravity," Adv. Theor. Math. Phys. 2, 231 (1998) [Int. J. Theor. Phys. 38, 1113 (1999)] [arXiv:hep-th/9711200].

[2] S. S. Gubser, I. R. Klebanov and A. M. Polyakov, "Gauge theory correlators from non-critical string theory," Phys. Lett. B 428, 105 (1998) [arXiv:hep-th/9802109].

[3] E. Witten, "Anti-de Sitter space and holography," Adv. Theor. Math. Phys. 2, 253 (1998) [arXiv:hep-th/9802150].

[4] C. P. Herzog, "Lectures on Holographic Superfluidity and Superconductivity," J. Phys. A A 42, 343001 (2009) [arXiv:0904.1975 [hep-th]].

[5] S. A. Hartnoll, P. K. Kovtun, M. Muller and S. Sachdev, "Theory of the Nernst effect near quantum phase transitions in condensed matter, and in dyonic black holes," Phys. Rev. B 76, 144502 (2007) [arXiv:0706.3215 [cond-mat.str-el]].

[6] H. Liu, J. McGreevy and D. Vegh, "Non-Fermi liquids from holography," Phys. Rev. D 83 (2011) 065029 [arXiv:0903.2477 [hep-th]].

[7] S. A. Hartnoll, "Lectures on holographic methods for condensed matter physics," Class. Quant. Grav. 26, 224002 (2009) [arXiv:0903.3246 [hep-th]].

[8] J. McGreevy, "Holographic duality with a view toward many-body physics," Adv. High Energy Phys. 2010, 723105 (2010) [arXiv:0909.0518 [hep-th]].

[9] S. Sachdev, "The Quantum phases of matter," arXiv:1203.4565 [hep-th].

[10] S. S. Gubser, "Breaking an Abelian gauge symmetry near a black hole horizon," Phys. Rev. D 78, 065034 (2008) [arXiv:0801.2977 [hep-th]].

[11] S. A. Hartnoll, C. P. Herzog and G. T. Horowitz, "Building a Holographic Superconductor," Phys. Rev. Lett. 101, 031601 (2008) [arXiv:0803.3295 [hep-th]].

[12] T. Nishioka, S. Ryu and T. Takayanagi, "Holographic Superconductor/Insulator Transition at Zero Temperature," JHEP 1003, 131 (2010) [arXiv:0911.0962 [hep-th]].

[13] S. S. Gubser, "Colorful horizons with charge in anti-de Sitter space," Phys. Rev. Lett. 101, 191601 (2008) [arXiv:0803.3483 [hep-th]].

[14] S. S. Gubser and S. S. Pufu, "The Gravity dual of a p-wave superconductor," JHEP 0811, 033 (2008) [arXiv:0805.2960 [hep-th]]. 
[15] Ryu, S., Hatsugai, Y. "Entanglement entropy and the Berry phase in the solid state," Phys. Rev. B 73, 245115 (2006) [arXiv:cond-mat/0601237].

[16] L. Amico, R. Fazio, A. Osterloh and V. Vedral, "Entanglement in many-body systems," Rev. Mod. Phys. 80, 517 (2008) [quant-ph/0703044 [QUANT-PH]].

[17] S. Ryu and T. Takayanagi, "Holographic derivation of entanglement entropy from AdS/CFT," Phys. Rev. Lett. 96, 181602 (2006) [hep-th/0603001].

[18] T. Nishioka, S. Ryu and T. Takayanagi, "Holographic Entanglement Entropy: An Overview," J. Phys. A A 42, 504008 (2009) [arXiv:0905.0932 [hep-th]].

[19] T. Takayanagi, "Entanglement Entropy from a Holographic Viewpoint," arXiv:1204.2450 [gr-qc].

[20] T. Albash and C. V. Johnson, "A Holographic Superconductor in an External Magnetic Field," JHEP 0809, 121 (2008) [arXiv:0804.3466 [hep-th]].

[21] S. A. Hartnoll, C. P. Herzog and G. T. Horowitz, "Holographic Superconductors," JHEP 0812, 015 (2008) [arXiv:0810.1563 [hep-th]].

[22] R. -G. Cai, L. Li, H. -Q. Zhang and Y. -L. Zhang, "Magnetic Field Effect on the Phase Transition in AdS Soliton Spacetime," Phys. Rev. D 84, 126008 (2011) [arXiv:1109.5885 [hep-th]].

[23] G. T. Horowitz and M. M. Roberts, "Holographic Superconductors with Various Condensates," Phys. Rev. D 78, 126008 (2008) [arXiv:0810.1077 [hep-th]].

[24] E. J. Brynjolfsson, U. H. Danielsson, L. Thorlacius and T. Zingg, "Holographic Superconductors with Lifshitz Scaling," J. Phys. A A 43, 065401 (2010) [arXiv:0908.2611 [hep-th]].

[25] R. -G. Cai and H. -Q. Zhang, "Holographic Superconductors with Horava-Lifshitz Black Holes," Phys. Rev. D 81, 066003 (2010) [arXiv:0911.4867 [hep-th]].

[26] G. T. Horowitz and M. M. Roberts, "Zero Temperature Limit of Holographic Superconductors," JHEP 0911, 015 (2009) [arXiv:0908.3677 [hep-th]].

[27] G. T. Horowitz, J. E. Santos and B. Way, "A Holographic Josephson Junction," Phys. Rev. Lett. 106, 221601 (2011) [arXiv:1101.3326 [hep-th]].

[28] M. Montull, O. Pujolas, A. Salvio and P. J. Silva, "Flux Periodicities and Quantum Hair on Holographic Superconductors," Phys. Rev. Lett. 107, 181601 (2011) [arXiv:1105.5392 [hep-th]].

[29] N. Bobev, A. Kundu, K. Pilch and N. P. Warner, "Minimal Holographic Superconductors from Maximal Supergravity," JHEP 1203, 064 (2012) [arXiv:1110.3454 [hep-th]].

[30] Y. Liu, Y. Peng and B. Wang, "Gauss-Bonnet holographic superconductors in BornInfeld electrodynamics with backreactions," arXiv:1202.3586 [hep-th].

[31] J. Erdmenger, P. Kerner and H. Zeller, "Transport in Anisotropic Superfluids: A Holographic Description," JHEP 1201, 059 (2012) [arXiv:1110.0007 [hep-th]]. 
[32] T. Albash and C. V. Johnson, "Holographic Studies of Entanglement Entropy in Superconductors," arXiv:1202.2605 [hep-th].

[33] R. -G. Cai, S. He, L. Li and Y. -L. Zhang, "Holographic Entanglement Entropy in Insulator/Superconductor Transition," arXiv:1203.6620 [hep-th].

[34] M. Ammon, J. Erdmenger, V. Grass, P. Kerner and A. O’Bannon, "On Holographic pwave Superfluids with Back-reaction," Phys. Lett. B 686, 192 (2010) [arXiv:0912.3515 [hep-th]].

[35] E. Witten, "Anti-de Sitter space, thermal phase transition, and confinement in gauge theories," Adv. Theor. Math. Phys. 2, 505 (1998) [hep-th/9803131].

[36] M. Srednicki, "Entropy and area," Phys. Rev. Lett. 71, 666 (1993) [hep-th/9303048].

[37] H. Casini and M. Huerta, "Entanglement and alpha entropies for a massive scalar field in two dimensions," J. Stat. Mech. 0512, P12012 (2005) [cond-mat/0511014].

[38] T. Nishioka and T. Takayanagi, "AdS Bubbles, Entropy and Closed String Tachyons," JHEP 0701, 090 (2007) [hep-th/0611035].

[39] B. Swingle and T. Senthil, "Universal crossovers between entanglement entropy and thermal entropy," arXiv:1112.1069 [cond-mat.str-el].

[40] T. Jacobson, "Black hole entropy and induced gravity," gr-qc/9404039.

[41] D. N. Kabat, "Black hole entropy and entropy of entanglement," Nucl. Phys. B 453, 281 (1995) [hep-th/9503016].

[42] S. N. Solodukhin, "Entanglement entropy of black holes and AdS/CFT correspondence," Phys. Rev. Lett. 97, 201601 (2006) [hep-th/0606205].

[43] R. Emparan, "Black hole entropy as entanglement entropy: A Holographic derivation," JHEP 0606, 012 (2006) [hep-th/0603081].

[44] N. Bobev, A. Kundu, K. Pilch and N. P. Warner, "Minimal Holographic Superconductors from Maximal Supergravity," JHEP 1203, 064 (2012) [arXiv:1110.3454 [hep-th]].

[45] S. S. Gubser, C. P. Herzog, S. S. Pufu and T. Tesileanu, "Superconductors from Superstrings," Phys. Rev. Lett. 103, 141601 (2009) [arXiv:0907.3510 [hep-th]].

[46] J. P. Gauntlett, J. Sonner and T. Wiseman, "Holographic superconductivity in MTheory," Phys. Rev. Lett. 103, 151601 (2009) [arXiv:0907.3796 [hep-th]].

[47] F. Aprile, D. Roest and J. G. Russo, "Holographic Superconductors from Gauged Supergravity," JHEP 1106, 040 (2011) [arXiv:1104.4473 [hep-th]].

[48] R. C. Myers and A. Singh, "Comments on Holographic Entanglement Entropy and RG Flows," JHEP 1204, 122 (2012) [arXiv:1202.2068 [hep-th]].

[49] H. Liu and M. Mezei, "A Refinement of entanglement entropy and the number of degrees of freedom," arXiv:1202.2070 [hep-th].

[50] I. R. Klebanov, D. Kutasov and A. Murugan, "Entanglement as a probe of confinement," Nucl. Phys. B 796, 274 (2008) [arXiv:0709.2140 [hep-th]]. 
[51] R. -G. Cai, Z. -Y. Nie and H. -Q. Zhang, "Holographic p-wave superconductors from Gauss-Bonnet gravity," Phys. Rev. D 82, 066007 (2010) [arXiv:1007.3321 [hep-th]].

[52] R. -G. Cai, Z. -Y. Nie and H. -Q. Zhang, "Holographic Phase Transitions of P-wave Superconductors in Gauss-Bonnet Gravity with Back-reaction," Phys. Rev. D 83, 066013 (2011) [arXiv:1012.5559 [hep-th]].

[53] J. de Boer, M. Kulaxizi and A. Parnachev, "Holographic Entanglement Entropy in Lovelock Gravities," JHEP 1107, 109 (2011) [arXiv:1101.5781 [hep-th]].

[54] L. -Y. Hung, R. C. Myers and M. Smolkin, "On Holographic Entanglement Entropy and Higher Curvature Gravity," JHEP 1104, 025 (2011) [arXiv:1101.5813 [hep-th]].

[55] N. Ogawa and T. Takayanagi, "Higher Derivative Corrections to Holographic Entanglement Entropy for AdS Solitons," JHEP 1110, 147 (2011) [arXiv:1107.4363 [hepth]].

[56] M. Ishihara, F. -L. Lin and B. Ning, "Refined Holographic Entanglement Entropy for the AdS Solitons and AdS black Holes," arXiv:1203.6153 [hep-th]. 